Interfaces and Free Boundaries 17 (2015), 55-68

DOI $10.4171 / \mathrm{IFB} / 333$

\title{
Obstacle mean-field game problem
}

\author{
Diogo A. Gomes \\ King Abdullah University of Science and Technology (KAUST), \\ CEMSE Division, Thuwal 23955-6900, Saudi Arabia, \\ and \\ KAUST SRI, Center for Uncertainty Quantification in \\ Computational Science and Engineering \\ E-mail:diogo.gomes@kaust.edu.sa \\ STEFANia PATRIzi \\ Weierstrass Institute for Applied Analysis and Stochastics \\ E-mail: stefaniapatrizi@yahoo.it
}

[Received 21 November 2013 and in revised form 12 October 2014]

\begin{abstract}
In this paper, we introduce and study a first-order mean-field game obstacle problem. We examine the case of local dependence on the measure under assumptions that include both the logarithmic case and power-like nonlinearities. Since the obstacle operator is not differentiable, the equations for first-order mean field game problems have to be discussed carefully. Hence, we begin by considering a penalized problem. We prove this problem admits a unique solution satisfying uniform bounds. These bounds serve to pass to the limit in the penalized problem and to characterize the limiting equations. Finally, we prove uniqueness of solutions.
\end{abstract}

2010 Mathematics Subject Classification: Primary 35J87; Secondary 49L99.

Keywords: Mean-field games; obstacle problem; penalization method.

\section{Introduction}

The mean-field game framework [21-26] is a class of methods that model the behavior of large populations of rational agents under a non-cooperative dynamic behavior. This research area has applications ranging from economics to engineering, as discussed in the recent surveys [5, 20, 27], the additional references therein, and the lectures by P. L. Lions in Collége de France [29].

In this paper, we investigate first-order mean-field game obstacle problems in the stationary periodic setting. To our knowledge, in the context of mean-field games, these problems were not studied previously. Before describing the problem, we start by recalling the original stationary meanfield game problem from [23], as well as the obstacle problem for Hamilton-Jacobi (H-J) equations [28].

Let $\mathbb{T}^{N}$ be the $N$-dimensional torus identified when convenient with $[0,1]^{N}$. Consider a continuous function, $H: \mathbb{R}^{N} \times \mathbb{T}^{N} \rightarrow \mathbb{R}$, the Hamiltonian, and a continuous increasing function, $g: \mathbb{R}_{0}^{+} \rightarrow \mathbb{R}$. In [23], the authors consider the stationary mean-field game system

$$
\left\{\begin{array}{l}
H(D u, x)=g(\theta)+\bar{H} \\
\operatorname{div}\left(D_{p} H \theta\right)=0,
\end{array}\right.
$$


where the unknowns are a function $u: \mathbb{T}^{N} \rightarrow \mathbb{R}$, a probability measure identified with its density $\theta: \mathbb{T}^{N} \rightarrow \mathbb{R}$ and a constant $\bar{H}$. The second equation is the adjoint of the linearization of the first equation in the variable $u$. This system (1.1) has the canonical structure of a mean-field game problem: a nonlinear elliptic or parabolic nonlinear partial differential equation (PDE) coupled with a PDE given by the adjoint of its linearization.

The existence of weak solutions for (1.1) was considered in [23]. In [10] mean-field games are not mentioned explicitly, however, the results there yield the existence of smooth solutions of (1.1) for $g(\theta)=\ln \theta$. The second order case, was also studied in [18] (see also [11]), [19], and [13]). Stationary mean-field games with congestion were considered in [12]. The time-dependent problem was addressed for parabolic mean-field games in [24], [8], [30], [17], [16], [14], [15], and in [6], and [7] for first-order mean-field games.

The first-order obstacle problem arises in optimal stopping (see [28], [2], [3], [1] and the references therein). In the periodic setting, a model problem is the following: let $\psi: \mathbb{T}^{N} \rightarrow \mathbb{R}$, and $H: \mathbb{T}^{N} \times \mathbb{R}^{N} \rightarrow \mathbb{R}$ be continuous functions. The obstacle problem is defined by

$$
\max \{H(D u, x), u-\psi(x)\}=0,
$$

where $u: \mathbb{T}^{N} \rightarrow \mathbb{R}$ is a bounded continuous function.

The linearization of the obstacle operator is not well-defined since the left-hand side of (1.2) may fail to be differentiable. Thus, it is not clear what should be the corresponding mean-field model. One of the contributions of this paper is the characterization of the appropriate analog to (1.1) for obstacle problems. This is achieved by applying the penalization method. This is a standard technique employed in many related problems, e.g. [28]. In the classical obstacle problem, to do so, one considers a family of smooth functions, $\beta_{\epsilon}: \mathbb{R} \rightarrow \mathbb{R}_{0}^{+}$, which vanish identically in $\mathbb{R}_{0}^{-}$and satisfy $\beta_{\epsilon}(z)=\frac{z-\epsilon}{\epsilon}$ for $z>\epsilon$. Then, obstacle problem is approximated by the equation

$$
H\left(D u_{\epsilon}, x\right)+\beta_{\epsilon}\left(u_{\epsilon}-\psi\right)=\epsilon \Delta u_{\epsilon}
$$

This equation admits viscosity solutions that satisfy uniform Lipschitz bounds. By sending $\epsilon \rightarrow 0$, one obtains a solution to (1.2).

Thanks to [28], for every $\epsilon>0$ there exists a smooth solution $u^{\varepsilon}$ to (1.3). It is also well known that, up to subsequences, $u^{\epsilon}$ converges uniformly to a viscosity solution $u$ of (1.2). The rate of convergence of this approximation was investigated using the nonlinear adjoint method in [4].

We then are led naturally to the approximate mean-field obstacle problem

$$
\left\{\begin{array}{l}
H\left(D u_{\epsilon}, x\right)+\beta_{\epsilon}\left(u_{\epsilon}-\psi\right)=g\left(\theta_{\epsilon}\right) \\
-\operatorname{div}\left(D_{p} H\left(D u_{\epsilon}, x\right) \theta_{\epsilon}\right)+\beta_{\epsilon}^{\prime}\left(u_{\epsilon}-\psi\right) \theta_{\epsilon}=\gamma(x) .
\end{array}\right.
$$

The additional term $\gamma$ in the right-hand side of (1.4) arises for the following reason: the mean-field obstacle problem models a population of agents trying to move optimally up to a certain stopping time at which they switch to the obstacle (the term $\beta_{\epsilon}^{\prime} \theta_{\epsilon}$ is the flow of agents switching to the obstacle). Without a source term introducing new agents in the system, we could fall into the pathological situation $\theta_{\epsilon} \equiv 0$. As it will be clear from the discussion, the approximate problem (1.4) admits smooth solutions even without additional elliptic regularization terms. This remarkable property is also true for certain first-order mean-field games, see, for instance, [9]. The function $u_{\epsilon}$ in (1.4) is the value function for an optimal stopping problem. This problem may not admit 
a continuous solution, [2], [3]. Owing to the structure of (1.4), we were able to prove regularity estimates that hold uniformly in $\epsilon$. However, in other related important situations, this may not be the case. It would be extremely interesting to consider a discontinuous viscosity solution approach for such problems.

As we will show in Section 4, by passing to the limit in (1.4), we obtain the mean-field obstacle problem

$$
\left\{\begin{array}{lll}
H(D u, x)=g(\theta) & \text { in } \quad \mathbb{T}^{N}, \\
-\operatorname{div}\left(D_{p} H(D u, x) \theta\right) \leqslant \gamma(x) & \text { in } \quad \mathbb{T}^{N}, \\
-\operatorname{div}\left(D_{p} H(D u, x) \theta\right)=\gamma(x) & \text { in } \quad\{u<\psi\} . \\
u \leqslant \psi . & &
\end{array}\right.
$$

This paper is structured as follows: after discussing the main hypothesis in Section 2, we prove, in Section 3, various estimates for (1.4) that are uniform in $\epsilon$. Namely, we obtain:

THEOREM 1.1 Under the assumptions of Section 2, let $\left(u_{\epsilon}, \theta_{\epsilon}\right)$ be the solution to (1.4). Then, there exists a constant $C$ independent of $\epsilon$ such that

$$
\begin{gathered}
\left\|u_{\epsilon}\right\|_{W^{2,2}\left(\mathbb{T}^{N}\right)} \leqslant C, \\
\left\|\theta_{\epsilon}\right\|_{\infty} \leqslant C, \\
\left\|\theta_{\epsilon}\right\|_{W^{1,2}\left(\mathbb{T}^{N}\right)} \leqslant C,
\end{gathered}
$$

and

$$
\left\|D u_{\epsilon}\right\|_{\infty} \leqslant C
$$

Applying these estimates, we consider the limit $\epsilon \rightarrow 0$ in Section 4. There we get the following result:

THEOREM 1.2 Under the assumptions of Section 2, let $\left(u_{\epsilon}, \theta_{\epsilon}\right)$ be the solution to (1.4). Then there exists $u \in W^{1, \infty}\left(\mathbb{T}^{d}\right) \cap W^{2,2}\left(\mathbb{T}^{d}\right)$, and $\theta \in L^{\infty}\left(\mathbb{T}^{d}\right) \cap W^{1,2}\left(\mathbb{T}^{d}\right)$ such that, through some subsequence,

$$
\begin{gathered}
u_{\epsilon} \rightarrow u \quad \text { in } L^{\infty}\left(\mathbb{T}^{N}\right), \\
D u_{\epsilon} \rightarrow D u, \quad \theta_{\epsilon} \rightarrow \theta \quad \text { in } L^{2}\left(\mathbb{T}^{N}\right), \\
D^{2} u_{\epsilon} \rightarrow D^{2} u \quad \text { in } L^{2}\left(\mathbb{T}^{N}\right),
\end{gathered}
$$

as $\epsilon \rightarrow 0$. Furthermore, $(u, \theta)$ solves (1.5).

Finally, in Section 5 we establish the uniqueness of solution of the limit problem. More precisely, our main result is:

THEOREM 1.3 Under the assumptions of Section 2, there exists a unique solution $(u, \theta) u \in$ $W^{1, \infty}\left(\mathbb{T}^{d}\right) \cap W^{2,2}\left(\mathbb{T}^{d}\right)$ and $\theta \in L^{\infty}\left(\mathbb{T}^{d}\right) \cap W^{1,2}\left(\mathbb{T}^{d}\right)$ of the mean-field obstacle problem (1.5).

\section{Assumptions}

In this section, we describe our main assumptions. First, to ease the presentation, we assume the obstacle to vanish, that is, $\psi \equiv 0$. This entails no loss of generality as we can always redefine the 
Hamiltonian and the solution so that the new obstacle vanishes. In addition, we will take the source term $\gamma(x)=1$. However, our results can be easily adapted to deal with a non-vanishing smooth source $\gamma$.

On the Hamiltonian $H$ and the function $g$ we assume:

(i) $H: \mathbb{R}^{N} \times \mathbb{R}^{N} \rightarrow \mathbb{R}$ is smooth and positive;

(ii) For each $p \in \mathbb{R}^{N}, x \rightarrow H(p, x)$ is periodic;

(iii) There exists a constant $\lambda>0$ such that

$$
H_{p_{i} p_{j}}(p, x) \xi_{i} \xi_{j} \geqslant \lambda|\xi|^{2}
$$

for all $p, x, \xi \in \mathbb{R}^{N}$;

(iv) There exists $C>0$ such that

$$
\begin{aligned}
& \left|D_{p p}^{2} H\right| \leqslant C \\
& \left|D_{x p}^{2} H\right| \leqslant C(1+|p|) \\
& \left|D_{x x}^{2} H\right| \leqslant C\left(1+|p|^{2}\right)
\end{aligned}
$$

and

$$
H(p, x)-D_{p} H(p, x) p \leqslant C
$$

for all $p, x \in \mathbb{R}^{N}$

(v) $g: \mathbb{R}^{+} \rightarrow \mathbb{R}$ is smooth and such that

(a) $g^{\prime}>0$,

(b) $g^{-1}(0)>0$,

(c) $\theta \rightarrow \theta g(\theta)$ is convex,

(d) there exist $C, \widetilde{C}>0$ and $\alpha \in\left[0, \alpha_{0}\right)$ with $\alpha_{0}$ the solution of

$$
2 \alpha_{0}=\left(\alpha_{0}+1\right) \beta(\beta-1), \quad \beta=\sqrt{\frac{2^{*}}{2}}
$$

if $N>2$, and $\alpha_{0}=\infty$ if $N \leqslant 2$, such that

$$
C \theta^{\alpha-1} \leqslant g^{\prime}(\theta) \leqslant \widetilde{C} \theta^{\alpha-1}+\widetilde{C},
$$

(e) for any $C_{0}>0$ there exists $C_{1}>0$ such that

$$
C_{0} \theta \leqslant \frac{1}{2} g(\theta) \theta+C_{1},
$$

for any $\theta \geqslant 0$.

We choose a penalization term $\beta_{\epsilon}: \mathbb{R} \rightarrow \mathbb{R}$, smooth, with $0 \leqslant \beta_{\epsilon}^{\prime} \leqslant \frac{1}{\epsilon}, \beta_{\epsilon}^{\prime \prime} \geqslant 0$ and such that

$$
\begin{gathered}
\beta_{\epsilon}(s)=0 \quad \text { for } s \leqslant 0, \quad \beta_{\epsilon}(s)=\frac{s-\epsilon}{\epsilon} \text { for } s>2 \epsilon \\
\left|\beta_{\epsilon}(s)-s \beta_{\epsilon}^{\prime}(s)\right| \leqslant C \text { for } s \in \mathbb{R} .
\end{gathered}
$$

REMARK 2.1 The typical examples we have in mind for $g$ are

$$
g(\theta)=\log (\theta)
$$


and

$$
g(\theta)=\theta^{\alpha}+\theta_{0}
$$

for some $\theta_{0}>0, \alpha \in\left(0, \alpha_{0}\right)$ with $\alpha_{0}$ as in Assumption 2.4.

REMARK 2.2 The assumptions on the Hamiltonian imply that

$$
\frac{\gamma}{2}|p|^{2}-C \leqslant H(p, x) \leqslant C|p|^{2}+C
$$

and

$$
\begin{aligned}
& \left|D_{p} H(p, x)\right| \leqslant C(1+|p|) \\
& \left|D_{x} H(p, x)\right| \leqslant C\left(1+|p|^{2}\right)
\end{aligned}
$$

for all $p, x \in \mathbb{R}^{N}$.

\section{A-priori estimates}

In this section, we will establish various a-priori estimates for smooth solutions of the approximate mean-field obstacle problem. Because these estimates will be uniform in $\epsilon$, we can pass to an appropriate limit as $\epsilon \rightarrow 0$, as explained in the next section.

In what follows, we denote by $(u, \theta)$ a classical solution of (1.4), and we will omit the subscript $\epsilon$ for convenience.

LEMMA 3.1 Under the assumptions of Section 2, there exist constants $C, \theta_{0}>0$ independent of $\epsilon$ such that for any solution $(u, \theta)$ of (1.4),

$$
\begin{gathered}
\theta \geqslant \theta_{0} \quad \text { in } \mathbb{T}^{N}, \\
\int_{\mathbb{T}^{N}} \theta d x \leqslant C, \\
\left|\int_{\mathbb{T}^{N}} \theta g(\theta) d x\right| \leqslant C, \\
\int_{\mathbb{T}^{N}}|u| d x \leqslant C,
\end{gathered}
$$

and

$$
\int_{\mathbb{T}^{N}}|D u|^{2} \theta d x \leqslant C .
$$

Proof. The lower bound on $\theta$ is a consequence of the fact that $g^{-1}$ is increasing with $g^{-1}(0)>0$, and $H$ and $\beta_{\epsilon}$ are non-negative:

$$
\theta=g^{-1}\left(H(D u, x)+\beta_{\epsilon}(u)\right) \geqslant g^{-1}(0)=: \theta_{0}>0 .
$$

Next, multiplying the first equation of (1.4) by $\theta$, the second equation by $u$, integrating and 
subtracting, we get

$$
\begin{aligned}
\int_{\mathbb{T}^{N}} g(\theta) \theta d x= & \int_{\mathbb{T}^{N}}\left(H(D u, x)+\beta_{\epsilon}(u)\right) \theta d x \\
= & \int_{\mathbb{T}^{N}}\left(H(D u, x)-D_{p} H(D u, x) D u\right) \theta d x \\
& \quad+\int_{\mathbb{T}^{N}}\left(\beta_{\epsilon}(u)-\beta_{\epsilon}^{\prime}(u) u\right) \theta d x+\int_{\mathbb{T}^{N}} u d x .
\end{aligned}
$$

Then, using (2.3) and (2.8), we can find a constant $C_{0}>0$ such that

$$
\int_{\mathbb{T}^{N}} g(\theta) \theta d x \leqslant C_{0} \int_{\mathbb{T}^{N}} \theta d x+\int_{\mathbb{T}^{N}} u d x \leqslant C_{0} \int_{\mathbb{T}^{N}} \theta d x+\int_{\mathbb{T}^{N}} u^{+} d x .
$$

Since, $g$ satisfies (2.6), we deduce that

$$
\frac{1}{2} \int_{\mathbb{T}^{N}} g(\theta) \theta d x \leqslant \int_{\mathbb{T}^{N}} u^{+} d x+C_{1} .
$$

Since $H \geqslant 0, \beta_{\epsilon}(u) \leqslant g(\theta)$. In particular, (3.7) implies

$$
\int_{\mathbb{T}^{N}} \beta_{\epsilon}(u) d x \leqslant \int_{\mathbb{T}^{N}} g(\theta) d x \leqslant \frac{1}{\theta_{0}} \int_{\mathbb{T}^{N}} g(\theta) \theta d x \leqslant C \int_{\mathbb{T}^{N}} u^{+} d x+C .
$$

At the same time, by (2.8),

$$
\int_{\mathbb{T}^{N}} \beta_{\epsilon}(u) d x \geqslant \int_{\{u>2 \epsilon\}} \beta_{\epsilon}^{\prime}(u) u d x-C=\frac{1}{\epsilon} \int_{\{u>2 \epsilon\}} u d x-C .
$$

Hence

$$
\frac{1}{\epsilon} \int_{\mathbb{T}^{N}} u^{+} d x \leqslant C \int_{\mathbb{T}^{N}} u^{+} d x+C,
$$

from which, for $\epsilon$ small enough, we get

$$
\int_{\mathbb{T}^{N}} u^{+} d x \leqslant C \epsilon .
$$

We infer, in particular, that $\int_{\mathbb{T}^{N}} g(\theta) \theta d x \leqslant C$ from which (3.2) follows. On the other hand, the convexity of $\theta g(\theta)$ implies

$$
\int_{\mathbb{T}^{N}} \theta g(\theta) d x \geqslant\left(\int_{\mathbb{T}^{N}} \theta d x\right) g\left(\int_{\mathbb{T}^{N}} \theta d x\right) \geqslant-C
$$

and (3.3) is then proven.

Estimate (3.4) can be proven observing that (3.6) combined with (3.2), (3.3), and estimate (3.8) yields

$$
\left|\int_{\mathbb{T}^{N}} u d x\right| \leqslant C
$$


This estimate, combined with (3.8) implies,

$$
\int_{\mathbb{T}^{N}} u^{-} d x \leqslant C
$$

from which then (3.4) follows.

Finally, using the first equation of (1.4) and (2.9) we get

$$
\int_{\mathbb{T}^{N}}|D u|^{2} \theta d x \leqslant C \int_{\mathbb{T}^{N}} g(\theta) \theta d x+C \int_{\mathbb{T}^{N}} \theta d x
$$

and then (3.5) is a consequence of (3.2) and (3.3).

LEMMA 3.2 Under the assumptions of Section 2, there exists a constant $C>0$ independent of $\epsilon$ such that for any solution $(u, \theta)$ of $(1.4)$

$$
\begin{gathered}
\|u\|_{W^{2,2}\left(\mathbb{T}^{N}\right)} \leqslant C, \\
\int_{\mathbb{T}^{N}} g^{\prime}(\theta)|D \theta|^{2} d x \leqslant C
\end{gathered}
$$

and

$$
\left\|\theta^{\frac{\alpha+1}{2}}\right\|_{W^{1,2}\left(\mathbb{T}^{N}\right)} \leqslant C
$$

Proof. Using (2.9), (3.1) and (3.3), we get

$$
\int_{\mathbb{T}^{N}}|D u|^{2} d x \leqslant C \int_{\mathbb{T}^{N}} H(D u, x) d x+C \leqslant C \int_{\mathbb{T}^{N}} g(\theta) d x+C \leqslant C \int_{\mathbb{T}^{N}} \theta g(\theta) d x+C \leqslant C .
$$

The previous bound on $\int_{\mathbb{T}^{N}}|D u|^{2} d x$, estimate (3.4), and the Poincaré inequality imply

$$
\|u\|_{L^{2}\left(\mathbb{T}^{N}\right)} \leqslant C .
$$

Next, differentiating twice with respect to $x_{i}$ the first equation in (1.4), and then summing on $i$ (we use Einstein's convention, that is, summing over repeated indices) we get

$$
\begin{aligned}
D_{p} H \cdot D(\Delta u)+\Delta_{x} H+2 H_{x_{i} p_{j}} u_{x_{j} x_{i}}+H_{p_{j} p_{l}} u_{x_{j} x_{i}} u_{x_{l} x_{i}} & \\
& +\beta_{\epsilon}^{\prime}(u) \Delta u+\beta_{\epsilon}^{\prime \prime}(u)|D u|^{2}=\Delta(g(\theta)) .
\end{aligned}
$$

Multiplying the previous equation by $\theta$ and using that

$$
\begin{aligned}
\int_{\mathbb{T}^{N}}\left(D_{p} H \cdot D(\Delta u)+\beta_{\epsilon}^{\prime}(u) \Delta u\right) \theta d x & =\int_{\mathbb{T}^{N}}\left(-\operatorname{div}\left(D_{p} H \theta\right)+\beta_{\epsilon}^{\prime}(u) \theta\right) \Delta u d x \\
& =\int_{\mathbb{T}^{N}} \Delta u d x=0,
\end{aligned}
$$

we obtain

$$
\begin{aligned}
\int_{\mathbb{T}^{N}}\left(\Delta_{x} H+2 H_{x_{i} p_{j}} u_{x_{j} x_{i}}+H_{p_{j} p_{l}} u_{x_{j} x_{i}} u_{x_{l} x_{i}}\right) \theta d x & \\
& =-\int_{\mathbb{T}^{N}} \beta_{\epsilon}^{\prime \prime}(u)|D u|^{2} \theta d x+\int_{\mathbb{T}^{N}} \Delta(g(\theta)) \theta d x .
\end{aligned}
$$


The uniformly convexity of $H$, properties (2.2), the convexity of $\beta_{\epsilon}$ and (3.5) then imply

$$
C \int_{\mathbb{T}^{N}}\left|D^{2} u\right|^{2} \theta d x+\int_{\mathbb{T}^{N}} g^{\prime}(\theta)|D \theta|^{2} d x \leqslant C \int_{\mathbb{T}^{N}}|D u|^{2} \theta d x+C \leqslant C
$$

which gives, in particular, (3.10). Moreover, from the previous inequality and (3.1), we infer that

$$
\int_{\mathbb{T}^{N}}\left|D^{2} u\right|^{2} d x \leqslant C
$$

This concludes the proof of (3.9). Finally, from (3.10) and (2.5) we infer that

$$
\int_{\mathbb{T}^{N}} \theta^{\alpha-1}|D \theta|^{2} d x \leqslant C \int_{\mathbb{T}^{N}} g^{\prime}(\theta)|D \theta|^{2} d x \leqslant C,
$$

that is $\left|D \theta^{\frac{\alpha+1}{2}}\right| \in L^{2}\left(\mathbb{T}^{N}\right)$. Since, in addition $\theta \in L^{1}\left(\mathbb{T}^{N}\right)$, the previous estimate and the Poincaré inequality imply that $\theta^{\frac{\alpha+1}{2}} \in L^{2}\left(\mathbb{T}^{N}\right)$ and so (3.11) holds.

We end this section with the proof of Theorem 1.1.

Proof of Theorem 1.1. Estimate (1.6) follows from lemma 3.2. Therefore, we proceed to prove the remaining bounds. First, we remark that from assumption (2.5), $g$ satisfies

$$
g(\theta) \leqslant C \theta^{\alpha}+C, \quad \text { when } \alpha>0,
$$

and

$$
g(\theta) \leqslant C \log (\theta)+C, \quad \text { when } \alpha=0 .
$$

Next, we show that $\beta_{\epsilon}^{\prime}(u)$ is bounded uniformly in $\epsilon$. The function $s \rightarrow \beta_{\epsilon}^{\prime}(s)$ is increasing. Hence $\beta_{\epsilon}^{\prime}(u)$ attains its maximum where $u$ has the maximum. Let $x_{0}$ be a maximum point of $u$, then $D u\left(x_{0}\right)=0$ and $D^{2} u\left(x_{0}\right) \leqslant 0$ and from (1.4), at $x=x_{0}$ we have

$$
\begin{aligned}
1 & =-\operatorname{div}\left(D_{p} H(D u, x) \theta\right)+\beta_{\epsilon}^{\prime}(u) \theta \\
& =-H_{p_{i} p_{j}} u_{x_{i} x_{j}} \theta-H_{p_{i} x_{i}} \theta-\frac{1}{g^{\prime}(\theta)}\left(u_{x_{i} x_{j}} H_{p_{i}} H_{p_{j}}+H_{x_{i}} H_{p_{i}}+\beta_{\epsilon}^{\prime}(u) u_{x_{i}} H_{p_{i}}\right)+\beta_{\epsilon}^{\prime}(u) \theta \\
& \geqslant-H_{p_{i} x_{i}} \theta-\frac{H_{x_{i}} H_{p_{i}}}{g^{\prime}(\theta)}+\beta_{\epsilon}^{\prime}(u) \theta .
\end{aligned}
$$

Then

$$
\max \beta_{\epsilon}^{\prime}(u)=\beta_{\epsilon}^{\prime}\left(u\left(x_{0}\right)\right) \leqslant H_{p_{i} x_{i}}\left(0, x_{0}\right)+\frac{H_{x_{i}}\left(0, x_{0}\right) H_{p_{i}}\left(0, x_{0}\right)}{g^{\prime}\left(\theta\left(x_{0}\right)\right) \theta\left(x_{0}\right)}+\frac{1}{\theta\left(x_{0}\right)} .
$$

Using the properties of the Hamiltonian, (2.5) and (3.1), we conclude that

$$
\max \beta_{\epsilon}^{\prime}(u) \leqslant C .
$$

Next, we claim that, for some constant $C$ independent on $p$,

$$
\int_{\mathbb{T}^{N}} \theta^{p-1}|D \theta|^{2} d x \leqslant C \int_{\mathbb{T}^{N}} \theta^{p+1}\left(1+|D u|^{4}\right) d x .
$$


In order to prove (3.15), we use the technique from [9] (see the proof of Theorem 5.1) and multiply equation the second equation in (1.4) by $\operatorname{div}\left(\theta^{p} D_{p} H(D u, x)\right)$, for $p>0$, and integrate by parts:

$$
\begin{aligned}
\int_{\mathbb{T}^{N}} \beta_{\epsilon}^{\prime} \theta \operatorname{div}\left(\theta^{p} D_{p} H\right) d x & =\int_{\mathbb{T}^{N}}\left(\theta H_{p_{i}}\right)_{x_{i}}\left(\theta^{p} H_{p_{j}}\right)_{x_{j}} d x \\
& =\int_{\mathbb{T}^{N}}\left(\theta H_{p_{i}}\right)_{x_{j}}\left(\theta^{p} H_{p_{j}}\right)_{x_{i}} d x \\
& =\int_{\mathbb{T}^{N}}\left(\theta\left(H_{p_{i}}\right)_{x_{j}}+\theta_{x_{j}} H_{p_{i}}\right)\left(\theta^{p}\left(H_{p_{j}}\right)_{x_{i}}+p \theta^{p-1} \theta_{x_{i}} H_{p_{j}}\right) d x \\
& =\int_{\mathbb{T}^{N}} \theta^{p+1}\left(H_{p_{i}}\right)_{x_{j}}\left(H_{p_{j}}\right)_{x_{i}}+p \theta^{p-1} H_{p_{i}} \theta_{x_{i}} H_{p_{j}} \theta_{x_{j}} \\
& =: \int_{\mathbb{T}^{N}} I_{1}+I_{2}+I_{3} d x . \theta^{p} \theta_{x_{i}} H_{p_{j}}\left(H_{p_{i}}\right)_{x_{j}} d x
\end{aligned}
$$

Using assumptions (2.2) on $H$, we get

$$
\begin{aligned}
I_{1} & =\theta^{p+1}\left(H_{p_{i} p_{k}} u_{x_{k} x_{j}}+H_{p_{i} x_{j}}\right)\left(H_{p_{j} p_{l}} u_{x_{l} x_{i}}+H_{p_{j} x_{i}}\right) \\
& \geqslant \theta^{p+1}\left[\gamma^{2}\left|D^{2} u\right|^{2}-C(1+|D u|)\left|D^{2} u\right|-C\left(1+|D u|^{2}\right)\right] \\
& \geqslant \theta^{p+1} \tilde{\gamma}^{2}\left|D^{2} u\right|^{2}-C \theta^{p+1}\left(1+|D u|^{2}\right),
\end{aligned}
$$

for some $\tilde{\gamma}>0$. Clearly

$$
I_{2}=p \theta^{p-1}\left|D_{p} H \cdot D \theta\right|^{2} .
$$

Let us estimate $I_{3}$ from below. From the first equation of (1.4), we gather that

$$
H_{p_{j}} u_{x_{j} x_{l}}=g^{\prime}(\theta) \theta_{x_{l}}-H_{x_{l}}-\beta_{\epsilon}^{\prime} u_{x_{l}} .
$$

Assumption (2.5) and the lower bound on $\theta$ (3.1), imply the existence of a positive constant $C_{0}$ such that

$$
g^{\prime}(\theta) \theta \geqslant C_{0}>0 .
$$

Then, using the properties of the Hamiltonian, (3.14), (3.17) and (3.18), we get

$$
\begin{aligned}
I_{3}= & (p+1) \theta^{p} \theta_{x_{i}} H_{p_{j}}\left(H_{p_{i} p_{l}} u_{x_{l} x_{j}}+H_{p_{i} x_{j}}\right) \\
= & (p+1) g^{\prime}(\theta) \theta^{p} H_{p_{i} p_{l}} \theta_{x_{i}} \theta_{x_{l}}+(p+1) \theta^{p} \theta_{x_{i}}\left(H_{p_{j}} H_{p_{i} x_{j}}-H_{p_{i} p_{l}} H_{x_{l}}\right) \\
& \quad-(p+1) \theta^{p} \beta_{\epsilon}^{\prime} H_{p_{i} p_{l}} \theta_{x_{i}} u_{x_{l}} \\
& \geqslant(p+1) \gamma C_{0} \theta^{p-1}|D \theta|^{2}-C(p+1) \theta^{p}|D \theta|\left(1+|D u|^{2}\right)-C(p+1) \theta^{p}|D \theta||D u| \\
\geqslant & =C(p+1) \theta^{p-1}|D \theta|^{2}-C(p+1) \theta^{p+1}\left(1+|D u|^{4}\right) .
\end{aligned}
$$

Next, let us bound from above the left-hand side of (3.16). We have

$$
\begin{aligned}
\beta_{\epsilon}^{\prime} \theta \operatorname{div}\left(\theta^{p} D_{p} H\right) & =\beta_{\epsilon}^{\prime} \theta\left(p \theta^{p-1} D_{p} H \cdot D \theta+\theta^{p} H_{p_{i} p_{j}} u_{x_{j} x_{i}}+\theta^{p} H_{p_{i} x_{i}}\right) \\
& \leqslant p \theta^{p-1}\left|D_{p} H \cdot D \theta\right|^{2}+\tilde{\gamma}^{2} \theta^{p+1}\left|D^{2} u\right|^{2}+C p \theta^{p+1}(1+|D u|),
\end{aligned}
$$

where, again, we used the properties of the Hamiltonian and (3.14). 
From the preceding estimates, we conclude that

$$
\begin{gathered}
C(p+1) \int_{\mathbb{T}^{N}} \theta^{p-1}|D \theta|^{2} d x-C(p+1) \int_{\mathbb{T}^{N}} \theta^{p+1}\left(1+|D u|^{4}\right) d x \\
\quad+p \int_{\mathbb{T}^{N}} \theta^{p-1}\left|D_{p} H \cdot D \theta\right|^{2} d x \\
\quad+\tilde{\gamma}^{2} \int_{\mathbb{T}^{N}} \theta^{p+1}\left|D^{2} u\right|^{2}-C \int_{\mathbb{T}^{N}} \theta^{p+1}\left(1+|D u|^{2}\right) d x \\
\leqslant \int_{\mathbb{T}^{N}} I_{1}+I_{2}+I_{3} d x \\
=\int_{\mathbb{T}^{N}} \beta_{\epsilon}^{\prime} \theta \operatorname{div}\left(\theta^{p} D_{p} H\right) d x \\
\leqslant p \int_{\mathbb{T}^{N}} \theta^{p-1}\left|D_{p} H \cdot D \theta\right|^{2} d x+\tilde{\gamma}^{2} \int_{\mathbb{T}^{N}} \theta^{p+1}\left|D^{2} u\right|^{2} d x \\
+C p \int_{\mathbb{T}^{N}} \theta^{p+1}(1+|D u|) d x .
\end{gathered}
$$

The previous inequalities imply (3.15).

By Lemma 3.2, if $N>2$, we have $\theta \in L^{\frac{2^{*}(1+\alpha)}{2}}$, and for $N=2, \theta \in L^{p}$, for all $p$. If $N=1$, the (1.7) holds trivially by Morrey's theorem.

Assume $N>2$, then Sobolev's inequality provides the bound

$$
\begin{aligned}
\left(\int_{\mathbb{T}^{N}} \theta^{\frac{p+1}{2} 2^{*}} d x\right)^{\frac{2}{2^{*}}} & \leqslant C \int_{\mathbb{T}^{N}} \theta^{p+1} d x+C \int_{\mathbb{T}^{N}}\left|D\left(\theta^{\frac{p+1}{2}}\right)\right|^{2} d x \\
& =C \int_{\mathbb{T}^{N}} \theta^{p+1} d x+C(p+1)^{2} \int_{\mathbb{T}^{N}} \theta^{p-1}|D \theta|^{2} d x .
\end{aligned}
$$

Let $\beta:=\sqrt{\frac{2^{*}}{2}}=\sqrt{\frac{N}{N-2}}>1$, then assumption (2.4) can be rewritten in the following way

$$
2 \alpha \leqslant(\alpha+1) \beta^{2} \frac{\beta-1}{\beta}
$$

and, for $\alpha>0$, it implies, together with (2.9) and (3.12) that

$$
|D u|^{4} \leqslant C(g(\theta))^{2}+C \leqslant C \theta^{2 \alpha}+C \leqslant C\left(1+\theta^{(\alpha+1) \beta^{2} \frac{\beta-1}{\beta}}\right) .
$$

The same inequality holds when $\alpha=0$, using (3.13):

$$
|D u|^{4} \leqslant C(g(\theta))^{2}+C \leqslant C(\log (\theta))^{2}+C \leqslant C\left(1+\theta^{(\alpha+1) \beta^{2} \frac{\beta-1}{\beta}}\right) .
$$


Therefore, from Hölder inequality we get

$$
\begin{aligned}
\int_{\mathbb{T}^{N}} \theta^{p+1}\left(1+|D u|^{4}\right) d x & \leqslant C \int_{\mathbb{T}^{N}} \theta^{p+1}\left(1+\theta^{(\alpha+1) \beta^{2} \frac{\beta-1}{\beta}}\right) d x \\
& \leqslant C \int_{\mathbb{T}^{N}} \theta^{p+1} d x+C\left(\int_{\mathbb{T}^{N}} \theta^{(p+1) \beta} d x\right)^{\frac{1}{\beta}}\left(\int_{\mathbb{T}^{N}} \theta^{(\alpha+1) \beta^{2}} d x\right)^{\frac{\beta-1}{\beta}} \\
& \leqslant C \int_{\mathbb{T}^{N}} \theta^{p+1} d x+C\left(\int_{\mathbb{T}^{N}} \theta^{(p+1) \beta} d x\right)^{\frac{1}{\beta}} \\
& \leqslant C\left(\int_{\mathbb{T}^{N}} \theta^{(p+1) \beta} d x\right)^{\frac{1}{\beta}} .
\end{aligned}
$$

The last inequality, (3.15) and (3.19) give the estimate

$$
\left(\int_{\mathbb{T}^{N}} \theta^{(p+1) \beta^{2}} d x\right)^{\frac{1}{\beta^{2}}} \leqslant C p^{2}\left(\int_{\mathbb{T}^{N}} \theta^{(p+1) \beta} d x\right)^{\frac{1}{\beta}} .
$$

Arguing as in [9], we get (1.7) and hence (1.9) for $N>2$.

When $N \leqslant 2$, the reasoning is similar, because $\theta \in L^{p}$ for any $p$, and so (1.7) holds too.

Finally, (1.8) is a consequence of (3.11) and the estimate (1.7) just proven.

\section{Convergence}

In this section, we present the proof of Theorem 1.2 using the previous estimates.

Proof of Theorem 1.2. Let $\left(u_{\epsilon}, \theta_{\epsilon}\right)$ be a solution of (1.4). The estimates obtained in the previous section, namely in Theorem 1.1, imply the existence of functions $u \in W^{2,2}\left(\mathbb{T}^{N}\right) \cap W^{1, \infty}\left(\mathbb{T}^{N}\right)$ and $\theta \in W^{1,2}\left(\mathbb{T}^{N}\right) \cap L^{\infty}\left(\mathbb{T}^{N}\right)$ such that, up to subsequence, as $\epsilon \rightarrow 0$

$$
\begin{gathered}
u_{\epsilon} \rightarrow u \quad \text { in } L^{\infty}\left(\mathbb{T}^{N}\right), \\
D u_{\epsilon} \rightarrow D u, \quad \theta_{\epsilon} \rightarrow \theta \quad \text { in } L^{2}\left(\mathbb{T}^{N}\right), \\
D^{2} u_{\epsilon} \rightarrow D^{2} u \quad \text { in } L^{2}\left(\mathbb{T}^{N}\right) .
\end{gathered}
$$

Furthermore, the sequence $u_{\epsilon}$ converges uniformly to a non-positive function $u$.

For $s>0$, since $\beta_{\epsilon}^{\prime}$ is increasing $\left(\beta_{\epsilon}^{\prime \prime}>0\right)$ and $\beta_{\epsilon}(0)=0$ we have

$$
\beta_{\epsilon}(s)=\beta_{\epsilon}(0)+\beta_{\epsilon}^{\prime}\left(\xi_{s}\right) s \leqslant \max _{t \in[0, s]} \beta_{\epsilon}^{\prime}(t) s=\beta_{\epsilon}^{\prime}(s) s .
$$

Therefore, for any $s \in \mathbb{R}$

$$
\beta_{\epsilon}(s) \leqslant \beta_{\epsilon}^{\prime}(s) s^{+} .
$$

This implies, using (3.14)

$$
0 \leqslant \beta_{\epsilon}\left(u_{\epsilon}\right) \leqslant \beta_{\epsilon}^{\prime}\left(u_{\epsilon}\right)\left(u_{\epsilon}\right)^{+} \leqslant C\left(u_{\epsilon}\right)^{+} .
$$


We conclude that $\beta_{\epsilon}\left(u_{\epsilon}\right) \rightarrow 0$ uniformly as $\epsilon \rightarrow 0$. Hence, the limit $(u, \theta)$ solves

$$
\begin{gathered}
H(D u, x)=g(\theta) \quad \text { in } \quad \mathbb{T}^{N}, \\
-\operatorname{div}\left(D_{p} H(D u, x) \theta\right) \leqslant 1 \quad \text { in } \quad \mathbb{T}^{N}, \\
-\operatorname{div}\left(D_{p} H(D u, x) \theta\right)=1 \quad \text { in }\{u<0\} . \\
u \leqslant 0 .
\end{gathered}
$$

\section{Uniqueness}

We end the paper with the proof of uniqueness of solutions to (1.5). This will be based upon a modified monotonicity argument inspired by the original technique by Lasry and Lions, see [23-25].

Proof of Theorem 1.3. Let $\left(u_{1}, \theta_{1}\right)$ and $\left(u_{2}, \theta_{2}\right)$ be distinct solutions of (1.5). Set

$$
A:=\left\{u_{1}-u_{2}>0\right\} .
$$

$A$ is an open set. Moreover, $A \subset\left\{u_{2}<0\right\}$ since $u_{1}-u_{2}=u_{1} \leqslant 0$ in $\left\{u_{2}=0\right\}$, therefore,

$$
-\operatorname{div}\left(D_{p} H\left(D u_{2}, x\right) \theta_{2}\right)=\gamma(x) \text { in } A .
$$

From the first equality in (1.5), we have

$$
\int_{A}\left[H\left(D u_{1}, x\right)-H\left(D u_{2}, x\right)\right]\left(\theta_{1}-\theta_{2}\right) d x=\int_{A}\left(g\left(\theta_{1}\right)-g\left(\theta_{2}\right)\right)\left(\theta_{1}-\theta_{2}\right) d x .
$$

Using the second inequality for $u_{1}$ and the third equality for $u_{2}$ in (1.5), multiplying by $u_{1}-u_{2}>0$ in $A$ and integrating by parts, we obtain

$$
\begin{aligned}
0 & \leqslant \int_{A} \operatorname{div}\left(D_{p} H\left(D u_{1}, x\right) \theta_{1}-D_{p} H\left(D u_{2}, x\right) \theta_{2}\right)\left(u_{1}-u_{2}\right) d x \\
& =-\int_{A}\left(D_{p} H\left(D u_{1}, x\right) \theta_{1}-D_{p} H\left(D u_{2}, x\right) \theta_{2}\right) D\left(u_{1}-u_{2}\right) d x .
\end{aligned}
$$

Note that there is no boundary data since $u_{1}-u_{2}=0$ on $\partial A$. Adding the two inequalities and using the convexity of $H$, we get

$$
\begin{aligned}
0 & \leqslant \int_{A}\left(g\left(\theta_{1}\right)-g\left(\theta_{2}\right)\right)\left(\theta_{1}-\theta_{2}\right) \\
& \leqslant \int_{A}\left[H\left(D u_{1}, x\right)-H\left(D u_{2}, x\right)\right]\left(\theta_{1}-\theta_{2}\right) d x \\
& \quad-\int_{A}\left(D_{p} H\left(D u_{1}, x\right) \theta_{1}-D_{p} H\left(D u_{2}, x\right) \theta_{2}\right) D\left(u_{1}-u_{2}\right) d x \\
& =-\int_{A}\left[H\left(D u_{2}, x\right)-H\left(D u_{1}, x\right)-D_{p} H\left(D u_{1}, x\right) D\left(u_{2}-u_{1}\right)\right] \theta_{1} d x \\
& \quad-\int_{A}\left[H\left(D u_{1}, x\right)-H\left(D u_{2}, x\right)-D_{p} H\left(D u_{2}, x\right) D\left(u_{1}-u_{2}\right)\right] \theta_{2} d x \\
& \leqslant-C \int_{A}\left|D\left(u_{1}-u_{2}\right)\right|^{2} d x .
\end{aligned}
$$


Thus we infer that $|A|=0$, i.e., $u_{1} \leqslant u_{2}$ almost everywhere.

Acknowledgement. D. Gomes was partially supported by KAUST baseline and start-up funds and KAUST SRI, Center for Uncertainty Quantification in Computational Science and Engineering.

\section{REFERENCES}

1. BARDi, M., \& CAPUZZO-DolcetTA, I., Optimal control and viscosity solutions of Hamilton-JacobiBellman equations, Birkhäuser Boston Inc., Boston, MA, 1997. With appendices by Maurizio Falcone and Pierpaolo Soravia. Zb10890.49011 MR1484411

2. Barles, G., \& Perthame, B., Discontinuous solutions of deterministic optimal stopping time problems, RAIRO Modél. Math. Anal. Numér. 21 (1987), 557-579. Zb10629. 49017 MR0921827

3. Barles, G., \& Perthame, B., Exit time problems in optimal control and vanishing viscosity method, SIAM J. Control Optim. 26 (1988), 1133-1148. Zbl0674. 49027 MR0957658

4. Cagnetti, F., Gomes, D., \& Tran, H. V., Adjoint methods for obstacle problems and weakly coupled systems of PDE, ESAIM, Control Optim. Calc. Var. 19 (2013), 754-779. Zb11273. 35090 MR3092361

5. Cardaliaguet, P., Notes on mean-field games.

6. Cardaliaguet, P., Long time average of first order mean-field games and weak kam theory, Dyn. Games Appl. 3 (2013) 473-488. Zbl06395224 MR3127145

7. Cardaliaguet, P., Weak solutions for first order mean-field games with local coupling, Preprint (2013).

8. Cardaliaguet, P., Lasry, J.-M., Lions, P.-L., \& Porretta, A., Long time average of mean field games, Netw. Heterog. Media 7 (2012), 279-301. Zb11270. 35098 MR2928380

9. Evans, L. C., Some new PDE methods for weak KAM theory., Calc. Var. Partial Differential Equations 17 (2003), 159-177. Zbl1032.37048 MR1986317

10. Evans, L. C., Further PDE methods for weak KAM theory, Calc. Var. Partial Differential Equations 35 (2009), 435-462. Zbl1179.35102 MR2496651

11. Gomes, D., Iturriaga, R., Sánchez-Morgado, H., \& YU, Y. Mather measures selected by an approximation scheme. Proc. Amer. Math. Soc. 138 (2010), 3591-3601. Zbl1204. 37063 MR2661558

12. Gomes, D., \& Mitake, H., Stationary mean-field games with congestion and quadratic hamiltonians, Preprint.

13. Gomes, D., Patrizi, S., \& Voskanyan, V., On the existence of classical solutions for stationary extended mean field games, Nonlinear Anal. 99 (2014), 49-79. Zbl1284. 49044 MR3160525

14. Gomes, D., \& Pimentel, E., Local regularity for mean-field games in the whole space, Minimax Theory and Applications.

15. Gomes, D., \& Pimentel, E. Time dependent mean-field games with logarithmic nonlinearities. Preprint.

16. Gomes, D., Pimentel, E., \& Sanchez-Morgado, H., Time dependent mean-field games in the superquadratic case, Comm. Partial Differential Equations 40 (2015), 40-76. MR3268921

17. Gomes, D., Pimentel, E., \& Sanchez-Morgado, H., Time dependent mean-field games in the subquadratic case, To appear in Comm. Partial Differential Equations (2014).

18. Gomes, D., \& SÁnchez Morgado, H., A stochastic Evans-Aronsson problem, Trans. Amer. Math. Soc. 366 (2014), 903-929. Zbl1286. 93203 MR3130321

19. Gomes, D. A., Pires, G. E., \& SÁncheZ-Morgado, H., A-priori estimates for stationary mean-field games, Netw. Heterog. Media 7 (2012), 303-314. Zbl1260. 49071 MR2928381

20. Gomes, D. A., \& SAÚde, J., Mean field games models - a brief survey, Dyn. Games Appl. 4 (2014), 110-154. Zb106395230 MR3195844 
21. Huang, M., Caines, P. E., \& Malhamé, R. P., Large-population cost-coupled LQG problems with nonuniform agents: individual-mass behavior and decentralized $\epsilon$-Nash equilibria, IEEE Trans. Automat. Control 52 (2007), 1560-1571. MR2352434

22. Huang, M., Malhamé, R. P., \& Caines, P. E., Large population stochastic dynamic games: closedloop McKean-Vlasov systems and the Nash certainty equivalence principle, Commun. Inf. Syst. 6 (2006), 221-251. Zbl1136.91349 MR2346927

23. LASRY, J.-M., \& LiOns, P.-L., Jeux à champ moyen. I. Le cas stationnaire, C. R. Math. Acad. Sci. Paris 343 (2006), 619-625. Zb11153.91009 MR2269875

24. LASRY, J.-M., \& LiOns, P.-L., Jeux à champ moyen. II. Horizon fini et contrôle optimal, C. R. Math. Acad. Sci. Paris 343 (2006), 679-684. Zbl1153. 91010 MR2271747

25. Lasry, J.-M., \& Lions, P.-L., Mean field games, Jpn. J. Math. 2 (2007), 229-260.

26. LASRY, J.-M., \& Lions, P.-L., Mean field games, Cahiers de la Chaire Finance et Développement Durable (2007).

27. Lasky, J.-M., Lions, P.-L., \& GuÉAnt, O., Mean field games and applications, Paris-Princeton lectures on Mathematical Finance (2010). Zbl1205.91027 MR2762362

28. Lions, P. L., Generalized solutions of Hamilton-Jacobi equations, vol. 69 of Research Notes in Mathematics, Pitman (Advanced Publishing Program), Boston, Mass., 1982.

29. LiONS, P.-L., College de france course on mean-field games.

30. Porretta, A., On the planning problem for the mean-field games system, Dyn. Games Appl. 4 (2014), 231-256. Zbl06395234 MR3195848 